\title{
Coherent Force Chains in Disordered Granular Materials Emerge from a Percolation of Quasilinear Clusters
}

\author{
K. P. Krishnaraj and Prabhu R. Nott \\ Department of Chemical Engineering, Indian Institute of Science, Bangalore 560012, India
}

(Received 5 July 2019; accepted 13 April 2020; published 12 May 2020)

\begin{abstract}
Dense granular materials and other particle aggregates transmit stress in a manner that belies their microstructural disorder. A subset of the particle contact network is strikingly coherent, wherein contacts are aligned nearly linearly and transmit large forces. Important material properties are associated with these force chains, but their origin has remained a puzzle. We classify subnetworks by their linear connectivity, and show the emergence of a percolation transition at a critical linearity at which the network is sparse, coherent, and contains the force chains. The subnetwork at critical linearity closely reflects the macroscopic stress and explains distinctive features of granular mechanics.
\end{abstract}

DOI: 10.1103/PhysRevLett.124.198002

Stress transmission in dense, amorphous aggregates of athermal particles, such as granular materials, emulsions, foams and biological cells, is characterized by a spatially inhomogeneous network of interparticle contact forces [1-10]. Numerous experimental [1-10] and computational 13-11]] studies have shown that a small subset of the force network, in the form of filamentary quasilinear structures called force chains, transmit large forces. The emergence of a seemingly ordered, spatially correlated network in a structurally disordered particle assembly with only shortranged repulsive interactions has been a long-standing puzzle. There is substantial evidence that this strong force subnetwork exerts significant influence on the mechanical and transport properties of the medium [10,12,14-18]understanding its origin and statistical properties is therefore of considerable value.

Previous studies that have analyzed force networks in granular materials have primarily aimed at identifying force chains, typically as clusters in which the normal force in all contacts exceeds a threshold $[4,5,11,12]$. More general classifications of connected clusters have also been attempted, by the use of techniques such as community detection [19] and topological network properties [20]. These studies point to the difficulty of objective identification of force chains based on pair interactions alone. More importantly, they do not offer an explanation for the origin of coherent force transmission in disordered media, and why the spatial correlation of force in force chains is long ranged.

Attempts to understand spatial correlation in granular force networks $[13,21]$ have used the normal contact force $F_{n}$ to classify subnetworks in grain assemblies subjected to isotropic compression. They found a percolation transition at a critical force $F_{n}^{c}$, with critical scaling in the vicinity of $F_{n}^{c}$, but came to contradictory conclusions: while Ref. [13] found the scaling exponents to differ from those of the random percolation universality class, and inferred the presence of long-ranged correlation in forces, the more recent study of Ref. [21] investigated larger systems and found the critical exponents to be almost the same as those of the random percolation universality class, thereby inferring the absence of long-ranged correlation. Moreover, these studies do not explain the linear structure of the force chains; indeed, we show in this Letter that the structure of the network at $F_{n}^{c}$ is nearly random, and bears little similarity with experimentally observed force chains.

In this study we use a network connectivity measure to classify subnetworks of connected contacts. Motivated by experimental observations [1-10] that force chains are roughly linearly aligned, we use a simple but robust definition of linearity as the connectivity measure. In computationally generated static granular assemblies subjected to a variety of external forcing, and in the dynamically forced system of steady shear, we find a percolation transition at a critical linearity at which the largest clusters span the system. We show that the clusters of critical linearity constitute the strong force network, of which force chains are a subset, and for which there is long-ranged spatial correlation of the contact force. The orientation of the clusters of critical linearity strongly reflects the imposed macroscopic stress, and explains distinctive, even anomalous, features of the stress in granular columns [22,23]. Finally, we show that linearity percolation is a generic feature of random geometric graphs, thereby explaining the prevalence of force chains in a variety of amorphous particulate systems.

The smallest connected network of particles is a triplet, the linearity of which is $r_{t}=\boldsymbol{n}_{1} \cdot \boldsymbol{n}_{2}$, where $\boldsymbol{n}_{1}$ and $\boldsymbol{n}_{2}$ are the unit normals at the two contacts. We define the linearity of a contact network as 


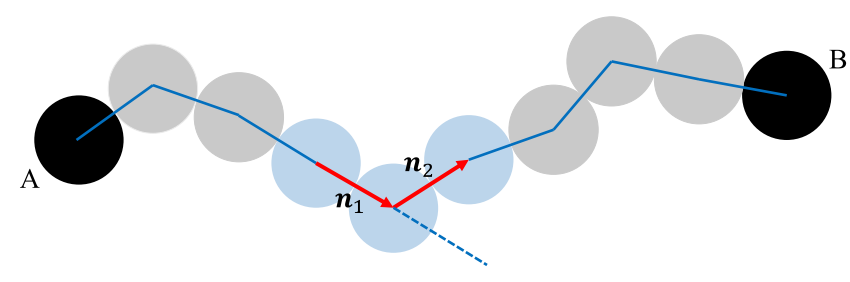

FIG. 1. Definition of cluster linearity. The linearity of the blue triplet is $r_{t}=\boldsymbol{n}_{1} \cdot \boldsymbol{n}_{2}$. The linearity $r$ of the cluster is the minimum of $r_{t}$ over all triplets in a connected cluster [Eq. (1)]. The end particles $\mathrm{A}$ and $\mathrm{B}$ are either at the boundaries, or in triplets of $r_{t} \leq 0$.

$$
r=\min \left(r_{t} \mid r_{t}>0\right)
$$

i.e., the minimum triplet linearity in the network such that the angle between adjacent normals is less than $\pi / 2$ (Fig. 1). It is intuitively apparent that this is a "weakest link" measure, as the fraction of the normal force from one contact that can be transmitted to the next decreases as $r_{t}$ approaches 0 . With this definition of connectivity, the network of particles in contact is transformed to a weighted graph, with the contacts being the nodes and their connections (triplets) being the edges (see Sec. II of Supplemental Material [24]). While this a purely configurational measure of connectivity, we show below that it captures the key features of the strong force network, sheds light on how it arises, and reveals its statistical features.

We generate configurations of a collection of spheres of mean diameter $d_{p}$ from computations using the discrete element method, which computes the motion of the particles using an elastoplastic interaction force. Slight polydispersity in size is maintained to avoid crystalline order. A variety of problems, corresponding to different boundary and forcing conditions were simulated in two and three dimensions. In each problem, the subnetwork of connected triplets of linearity $r$ is sieved out of the entire network, and its connectivity characterized by enumerating the number clusters with $s$ contacts $n(s, r)$ using tools from graph analysis. Details of the computational method, generation of configurations, subnetwork sampling, and the determination of network statistics are given in the Supplemental Material [24]. All the results reported are averages over a large number of configurations.

To demonstrate linearity percolation, we first consider a system of $N$ particles in a two-dimensional square domain of size $L$ subjected to isotropic compression. When $r$ is near unity, there are only isolated triplets; as $r$ is decreased, the fraction of connected triplets remains small until a critical linearity $r_{c}$, at which connected triplets percolate through the system [Fig. 2(a)]. The percolation transition is robust - it is observed for a wide range of pressure and particle interaction parameters (Fig. S3.1c and Sec. V in the Supplemental Material [24]). The transition exhibits the scaling properties of bond percolation and continuous phase transitions [25]: in the vicinity of $r_{c}$ the percolation
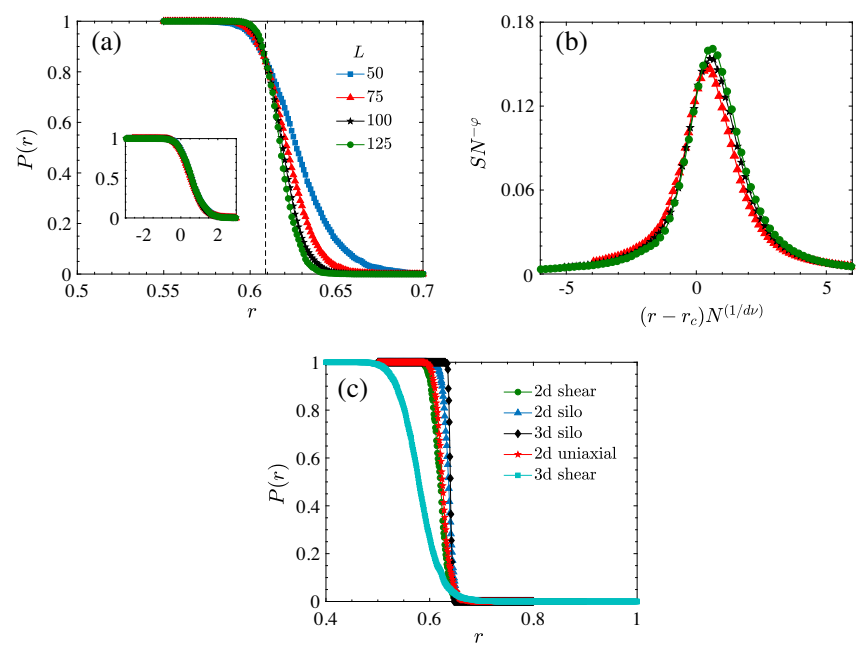

FIG. 2. Linearity percolation. (a) Percolation probability $P(r)$ for $2 \mathrm{D}$ isotropic compression for different system sizes $L$ (in units of $d_{p}$ ), the inset showing the collapse for different system sizes when the abscissa is plotted as $\left(r-r_{c}\right) N^{1 / d \nu}$. The dashed line marks the value of $r_{c}$, which here is 0.609 ; it is in general a function of the area fraction. (b) Finite size scaling of the mean cluster size $S(r)$. (c) Percolation probability $P(r)$ for anisotropic forcing, in two and three dimensions. The geometries and boundary conditions are described in Sec. III of the Supplemental Material [24].

probability $P(r)$ varies as $f\left[\left(r-r_{c}\right) N^{1 / d \nu}\right]$, and the mean cluster size $S(r)$ varies as $N^{\varphi} g\left[\left(r-r_{c}\right) N^{1 / d \nu}\right]$, where $\varphi, \nu$ are the critical exponents, and $d$ is the dimension of the system [Fig. 2(b)]. The values of $r_{c}$ and the exponents are obtained using standard techniques (see Sec. II. B of the Supplemental Material [24]): our estimates are $\varphi=0.93 \pm$ 0.29 and $\nu=1.15 \pm 0.35$. We note that the uncertainties in the estimates of $\varphi$ and $\nu$ are significant, indicating that averaging over more configurations is necessary (see Sec. II. B of the Supplemental Material [24]), which is currently computationally prohibitive. This precludes a conclusion on whether or not the network belongs to the random percolation university class [25] $(\varphi=43 / 48$, $\nu=4 / 3$ ), but we show below that this has no bearing on the nature of force correlation. It is noteworthy that for the same system, the linearity of force percolated clusters discussed earlier with reference to Refs. $[13,21]$ is less than 0.1 , as shown on Fig. S2.2 of the Supplemental Material [24]; the figure also shows, for three randomly chosen configurations, that the maximally linear force percolating clusters (with force cutoff of $F_{n}^{c}$ ) are much more tortuous than linearity percolating clusters. It is thus clear that the clusters at $F_{n}^{c}$ are virtually random, and bear little resemblance to force chains.

Importantly, we observe linearity percolation even for anisotropic forcing, such as static grain assemblies subjected to uniaxial compression and gravity-bound silos, and in the dynamically forced system of steady shear [Fig. 2(c)]. This shows that such a connectivity transition is 

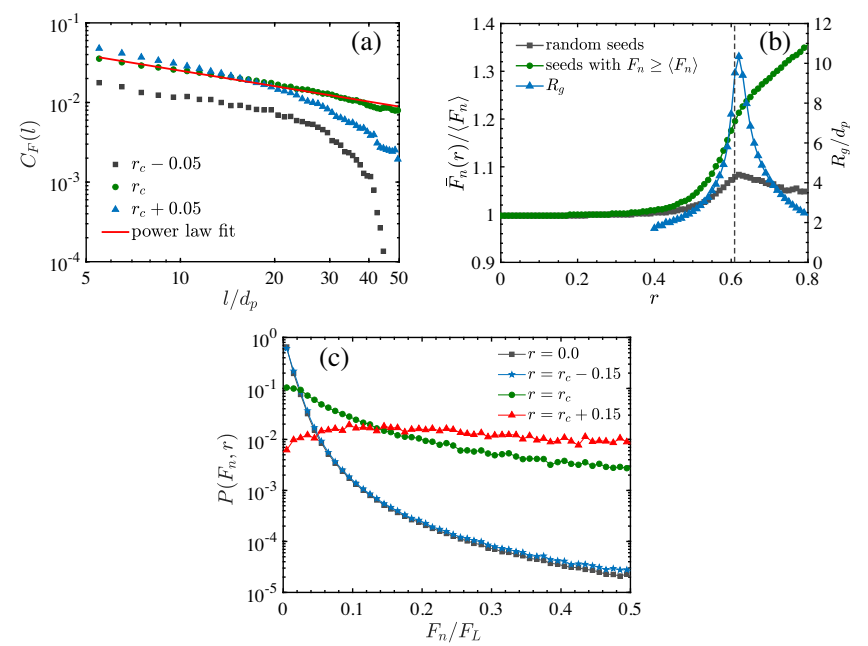

FIG. 3. Statistics of contact forces in subnetworks of different linearity. (a) Spatial correlation function of the contact force $C_{F}(\ell)$ in the vicinity of critical linearity; the red line is the power law fit $a \ell^{b}$ at critical linearity ( $a=0.11, b=-0.65$ ). (b) Variation of average contact force with linearity $r$, for random seeds (black squares) and seeds with a minimum contact force of the global mean $\left\langle F_{n}\right\rangle$ (green circles). The radius of gyration $R_{g}$ of nonpercolating clusters is also shown. The dashed line marks critical linearity $r_{c}=0.609$. The results in $\mathbf{a}$ and $\mathbf{b}$ are for $2 \mathrm{D}$ isotropic compression with system size $L=100 d_{p}$. (c) Probability distribution of the incremental normal contact force due to a point force $F_{L}$ acting on the surface of a 2D rectangular bed under gravity (see Sec. III. D in the Supplemental Material [24]), in clusters of different linearity; here $r_{c}$ is 0.66 .

a general signature of granular microstructure in static and slowly deforming states. Estimating the critical exponents for anisotropic systems is not straightforward, and not well studied. However, for our analysis it suffices to obtain an estimate of $r_{c}$ from the maximum of $\bar{F}_{n}(r)$, defined below (see Fig. S2.1 of the Supplemental Material [24]); as shown in Fig. 3(b), this provides a good estimate for isotropic compression. We show presently that this estimate of $r_{c}$ usefully connects the network structure to the macroscopic stress.

Though $r$ is a purely configurational quantity and its percolation is determined without reference to the forces in the contact network, the spatial correlation of force in the network depends strongly on it. To demonstrate this, we compute the correlation function $C_{F}(l)=$ $\left\langle\delta\left(l_{i j}-l\right) F_{n_{i}}^{\prime} F_{n_{j}}^{\prime}\right\rangle$, where $F_{n_{i}}^{\prime}$ is the deviation of the normal force at contact $i$ from the mean over all pairs $\left\langle F_{n}\right\rangle, l_{i j}$ is the distance between contacts $i$ and $j$, and the angle brackets denote averaging over the subnetwork of linearity $r$ over multiple configurations. $C_{F}(l)$ exhibits a power law decay at $r=r_{c}$ and an exponential decay above or below $r_{c}$ [Fig. 3(a)], indicating long-ranged correlation at critical linearity, and short-ranged correlation away from it. The mechanical relevance of linearity becomes clearer when we consider the mean force $\bar{F}_{n}(r)$ in the subnetwork of linearity $r$-we see that $\bar{F}_{n}(r)$ is maximum at $r_{c}$ [Fig. 3(b)]. The peak is clearly discernible, but small because the network is sampled from seed contacts chosen randomly; if the seeds are chosen from the subset of contacts that bear a normal force of at least $\left\langle F_{n}\right\rangle$, the mean force in the subnetwork rises much more sharply as $r$ increases to $r_{c}$, followed by a linear rise above $r_{c}$. The rise in $\bar{F}_{n}$ for $r>r_{c}$ is not of mechanical significance, as the radius of gyration of connected clusters drops sharply above $r_{c}$, and so does their occurrence (see Fig. S2.4 of the Supplemental Material [24]). Thus, clusters of high linearity do not necessarily bear large forces, but the large force bearing clusters are of linearity $r_{c}$. The fraction of particles in the subnetwork at linearity $r_{c}$ is small, as shown in Fig. S2.4 of the Supplemental Material [24] - it is now clear that the subnetwork at critical linearity is sparse, coherent, and contains the force chains.

A statistical feature of forces in grain assemblies that has been widely observed $[2,3,12,26]$ is that the probability distribution of normal contact force $P\left(F_{n}\right)$ decays exponentially for large $F_{n}$. In the context of our analysis, it is useful to determine the contact force distribution $P\left(F_{n}, r\right)$ in subnetworks of linearity $r$. This is best illustrated by considering the incremental force network arising from a point force $F_{L}$ applied on the surface of a gravity-deposited granular bed. The distributions for different $r$ are shown in Fig. 3(c), where it is clear that the probability of finding a large contact force is much higher in subnetworks of linearity $r_{c}$ : specifically, $P\left(\frac{1}{2} F_{L}, r_{c}\right)$ is over 100 times larger than $P\left(\frac{1}{2} F_{L}, 0\right)$. The results in Fig. 3 provide clear evidence that force chains are subsets of critically linear clusters.

Experiments and simulations have shown that force chains in $2 \mathrm{D}$ assemblies tend to align along the direction of major principal stress $[3,12,27]$. To probe this feature, we consider the probability distribution $P_{\mathrm{cl}}(\theta, r)$ of the orientation of clusters in networks of different linearity (see Sec. II. D of the Supplemental Material [24]). For isotropic compression, $P_{\mathrm{cl}}(\theta, r)$ too is isotropic, irrespective of $r$ [Fig. 4(a)]. In uniaxial compression and plane shear, $P_{\mathrm{cl}}\left(\theta, r_{c}\right)$ has sharp peaks in the directions of the major principal stress [Fig. 4(b), 4(c)], but the distribution is nearly isotropic for $r$ even slightly less than $r_{c}$-thus, only the subnetwork at $r_{c}$ closely reflects the anisotropy of forcing. Most previous studies have quantified microstructural anisotropy by examining the distribution of the pair contact vector $\boldsymbol{n}$ (Fig. 1), but it is clear from Fig. 4(b), 4(c) that the orientation of critical clusters is a much more accurate indicator of the macroscopic forcing.

Indeed, the orientation of critical clusters explains some distinctive and non-trivial features of the stress in granular columns under gravity. It is well known that the stress in a static vertical column of grains saturates exponentially with depth, as a result of a vertical shear stress $\sigma_{y z}$ imposed by the walls owing to Coulomb friction [22,28]; this effect has also been observed in a horizontal layer of disks driven by a 
(a)

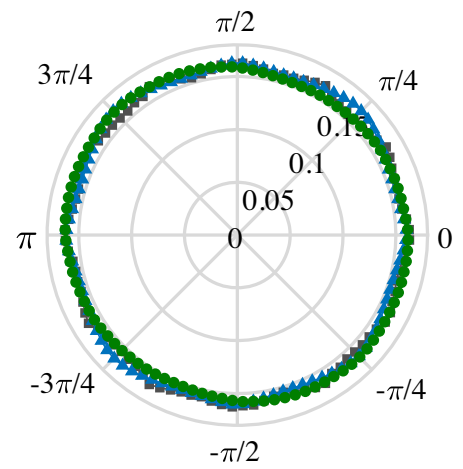

(b)

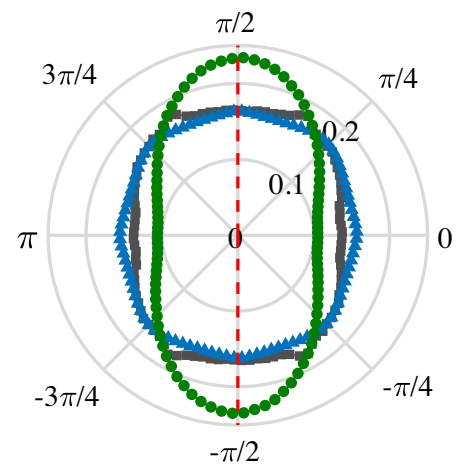

(c)

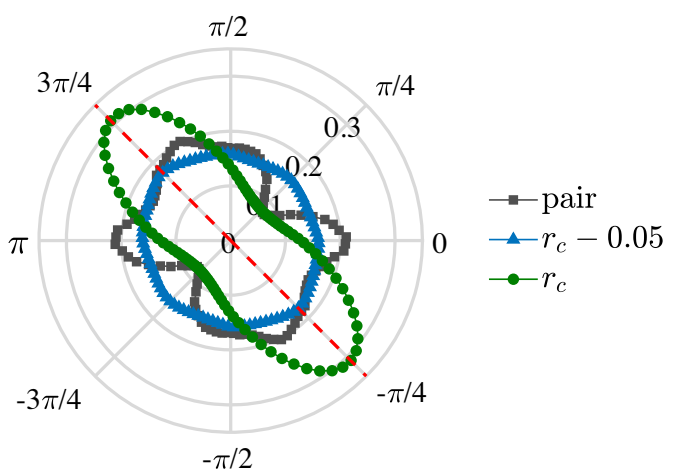

(d)

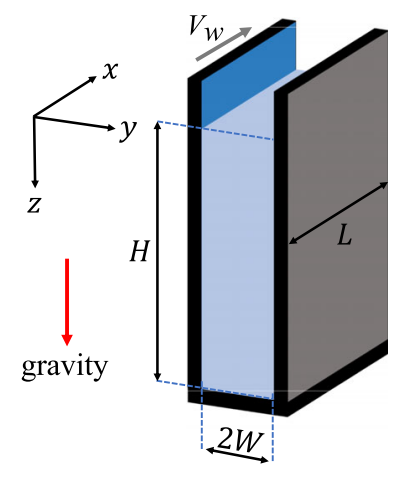

(e)

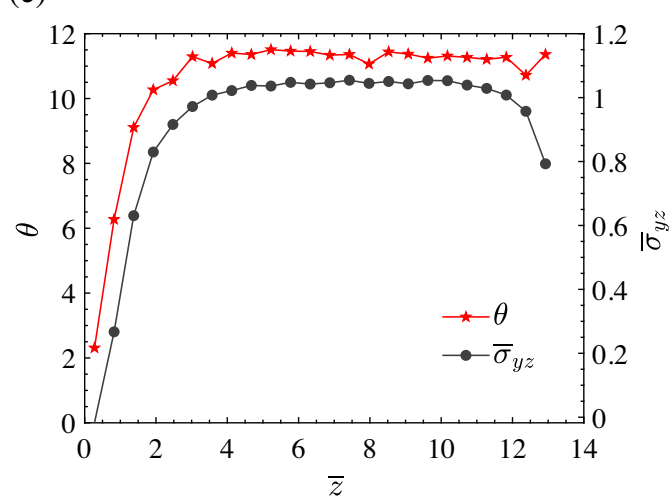

(f)

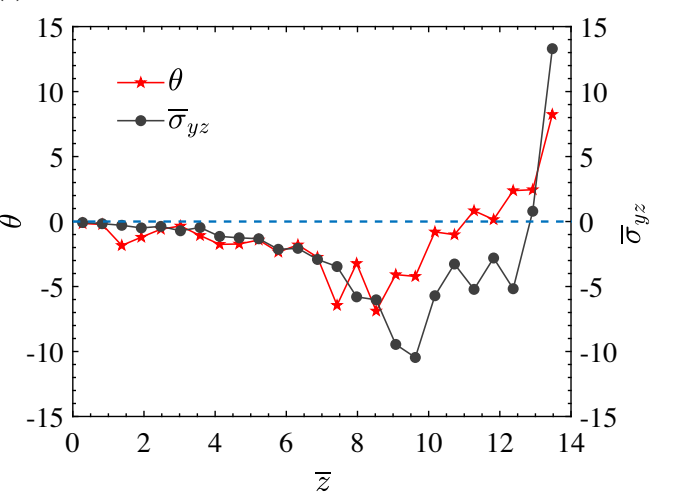

FIG. 4. Clusters of critical linearity reflect the macroscopic stress. (a) Orientation distributions of clusters of different linearity, $P_{\mathrm{cl}}(\theta, r)$, and the pair contact vector for different forcing: (a) Isotropic compression $\left(r_{c}=0.6089\right)$, (b) uniaxial compression $\left(r_{c}=0.63\right)$, and (c) plane shear $\left(r_{c}=0.62\right)$. In (b) and (c) the red dashed lines indicate the principal directions of compression. (d) Schematic of a 3D granular column; the column is static when the velocity $V_{w}$ is zero, and it is sheared in the horizontal direction when $V_{w}$ is nonzero. (e),(f) Variation of the mean critical cluster angle [see Fig. S2.3(b) [24] ] and axial shear stress with depth $z$ in a 3D granular column of lateral dimension $2 W=20 d_{p}$ when the column is, respectively, static, and sheared in the $y$ direction. Here, $\bar{z} \equiv z /(2 W)$ and $\bar{\sigma}_{y z} \equiv \sigma_{y z} /(\rho g W)$ are the scaled depth and vertical shear stress, where $\rho$ is the bulk density of the granular material in the column, and $g$ the gravitational acceleration.

frictional belt, provided the confining lateral walls have a sawtooth serration [29]. Recently, a curious feature was revealed when the vertical column is sheared in the horizontal direction [23] by moving one wall relative to the other with velocity $V_{w}$ [Fig. 4(d)]; it was observed that $\sigma_{y z}$ changes sign due to a dilation-driven secondary flow [30], and the magnitudes of all stress components rise exponentially with depth. In both these cases, we trace out clusters of critical linearity starting from seed contacts on the walls, and examine their average orientation $\theta$ (see Sec. II. D of the Supplemental Material [24]). In a static column, $\theta$ is positive [Fig. 4(e)], indicating that the clusters transmit a downward traction to the walls, or $\sigma_{y z}>0$. In a sheared column, $\theta$ is negative [Fig. 4(f)], indicating that the critical clusters transmit an upward traction to the walls, whence $\sigma_{y z}<0$. In both cases, the variation of $\sigma_{y z}$ with depth is closely reflected by that of $\theta$. Thus, the intriguing observation of Ref. [30], which is yet to find a complete continuum mechanical explanation, is reflected in the orientation of the clusters of critical linearity.
Furthermore, in the sheared column the static base influences the stress at sufficiently large depths $(\bar{z}>10)$, causing $\sigma_{y z}$ to change sign; this too is reflected in the profile of $\theta$ [Fig. 4(f)].

It is pertinent to ask what aspect of grain interactions leads to percolation of linearity? To answer this question, we studied the connectivity of random geometric graphs [31,32] (see Sec. IV of the Supplemental Material [24]), wherein edges (contacts) are connected randomly, but keeping the average number of connections per node (particle) fixed. Interestingly, we find linearity percolation in such a graph, with critical exponents for the percolation transition $\varphi=0.98 \pm 0.034, \nu=1.42 \pm 0.037$ that differ considerably from those of the random universality class. The critical exponents fall within the range of uncertainty of our estimates for particle configurations generated by isotropic compression, discussed earlier. It is well known that for a fixed spatial dimension, the critical exponents are independent of the lattice structure. This suggests strongly that linearity percolation, and thereby force chains, arise 
from topological constraints of the contact network, rather than the details of the interaction force (such as friction) and the balance of force and torque on each particle, as suggested by some studies $[4,5,13]$. It is also a plausible explanation for why similar force networks are observed in a variety of other aggregates of athermal particles, such as emulsions, foams, and living cells [1-9].

In conclusion, we have shown that coherent transmission of force via force chains in disordered granular materials arises from a percolation of quasilinear clusters. The subnetwork at critical linearity, corresponding to the percolation transition, exhibits most of the mechanical and statistical features commonly associated with dense granular materials $[3,12,22,23,27]$, thereby elucidating the importance of force chains in granular mechanics. Our results throw light on why force chains are seen in many disparate physical systems [1-9]. Furthermore, we have shown that the configuration of particle positions determines the nature of force transmission-this is an important result, as it is commonly believed that for a system of jammed particles, the configuration of interaction forces is a more appropriate descriptor of its state, as a very small perturbation in a particle's position can cause a large change in its interaction forces. While this is certainly true at the particle scale, our study shows that the position configuration at large scales, or the topology of the contact network, determines the mechanics. Our study makes two important connections to current studies on dense particulate materials. The first relates to continuum models, where the need for introducing a fabric tensor in the constitutive relation for the stress $[23,33,34]$ has been increasingly felt. While all previous studies have used the pair contact vector to derive a fabric, we make a compelling case for using a fabric based on the orientation of clusters of critical linearity. The second connection relates to the statistics of particle configurations: a long-standing proposal [35] is that all configurations for a fixed volume (that satisfy the constraints of force balance on particles) are equally probable, which has found some computational validation $[36,37]$. Our results indicate that even if this proposal is valid, only a small subset of the particle assembly in each configuration, which corresponds to linearity percolating clusters, are of mechanical relevance. Sorting configurations based on the linearity of percolating clusters therefore appears to be a worthwhile pursuit, which we are currently working on.

This work was funded by the Science and Engineering Research Board under Grant No. EMR/2016/002817. K.P.K. acknowledges funding from the Ministry of Human Resources Development, India. We acknowledge useful discussions with Sriram Ramaswamy and Bulbul Chakraborty. P. R. N. acknowledges discussions with
Emanuela del Gado, Peter Olmsted, and several other participants in a programme held at the Kavli Institute for Theoretical Physics, which was supported by the National Science Foundation under Grant No. NSF PHY-1748958.

*Corresponding author. prnott@iisc.ac.in

[1] P. Dantu, in Proceedings of the 4th International Conference on Soil Mechanics and Foundations Engineering (Butterworths, London, 1957), pp. 144-148.

[2] C. H. Liu, S. R. Nagel, D. A. Schecter, S. N. Coppersmith, S. Majumdar, O. Narayan, and T. A Witten, Science 269, 513 (1995).

[3] T. S. Majmudar and R. P. Behringer, Nature (London) 435, 1079 (2005).

[4] K. W. Desmond, P. J. Young, D. Chen, and E. R. Weeks, Soft Matter 9, 3424 (2013).

[5] J. Zhou, S. Long, Q. Wang, and A. D. Dinsmore, Science 312, 1631 (2006).

[6] J. Brujić, S. F. Edwards, D. V. Grinev, I. Hopkinson, D. Brujić, and H.A. Makse, Faraday Discuss. 123, 207 (2003).

[7] N. Brodu, J. A. Dijksman, and R. P. Behringer, Nat. Commun. 6, 6361 (2015).

[8] G. Katgert and M. van Hecke, Europhys. Lett. 92, 34002 (2010).

[9] M. Delarue, J. Hartung, C. Schreck, P. Gniewek, L. Hu, S. Herminghaus, and O. Hallatschek, Nat. Phys. 12, 762 (2016).

[10] D. Howell, R. P. Behringer, and C. Veje, Phys. Rev. Lett. 82, 5241 (1999).

[11] J. F. Peters, M. Muthuswamy, J. Wibowo, and A. Tordesillas, Phys. Rev. E 72, 041307 (2005).

[12] F. Radjai, D. E. Wolf, M. Jean, and J. J. Moreau, Phys. Rev. Lett. 80, 61 (1998).

[13] S. Ostojic, E. Somfai, and B. Nienhuis, Nature (London) 439, 828 (2006).

[14] H. A. Makse, D. L. Johnson, and L. M. Schwartz, Phys. Rev. Lett. 84, 4160 (2000).

[15] R. C. Hidalgo, C. U. Grosse, F. Kun, H. W. Reinhardt, and H. J. Herrmann, Phys. Rev. Lett. 89, 205501 (2002).

[16] E. T. Owens and K. E. Daniels, Europhys. Lett. 94, 54005 (2011).

[17] N. Vandewalle, C. Lenaerts, and S. Dorbolo, Europhys. Lett. 53, 197 (2001).

[18] A. Smart, P. Umbanhowar, and J. Ottino, Europhys. Lett. 79, 24002 (2007).

[19] D. S. Bassett, E. T. Owens, M. A. Porter, M. L. Manning, and K. E. Daniels, Soft Matter 11, 2731 (2015).

[20] M. Kramar, A. Goullet, L. Kondic, and K. Mischaikow, Phys. Rev. E 87, 042207 (2013).

[21] S. N. Pathak, V. Esposito, A. Coniglio, and M. P. Ciamarra, Phys. Rev. E 96, 042901 (2017).

[22] H. A. Janssen, Z. Ver. Dtsch. Ing. 39, 1045 (1895).

[23] V. Mehandia, K. J. Gutam, and P. R. Nott, Phys. Rev. Lett. 109, 128002 (2012). 
[24] See Supplemental Material at http://link.aps.org/ supplemental/10.1103/PhysRevLett.124.198002 for additional text and figures that provide details of the computational method, the protocol for generation of configurations, the tools used for subnetwork sampling, and the determination of network statistics.

[25] D. Stauffer and A. Aharony, Introduction to Percolation Theory (Taylor \& Francis, London, 2014).

[26] E. I. Corwin, H. M. Jaeger, and S. R. Nagel, Nature (London) 435, 1075 (2005).

[27] F. da Cruz, S. Emam, M. Prochnow, J. N. Roux, and F. Chevoir, Phys. Rev. E 72, 021309 (2005).

[28] K. K. Rao and P. R. Nott, An Introduction to Granular Flow (Cambridge University Press, New York, 2008).
[29] M. Y. Karim and E. I. Corwin, Phys. Rev. Lett. 112, 188001 (2014).

[30] K. P. Krishnaraj and P. R. Nott, Nat. Commun. 7, 10630 (2016).

[31] J. Dall and M. Christensen, Phys. Rev. E 66, 016121 (2002).

[32] M. Barthélemy, Phys. Rep. 499, 1 (2011).

[33] J. Sun and S. Sundaresan, J. Fluid Mech. 682, 590 (2011).

[34] R. N. Chacko, R. Mari, S. M. Fielding, and M. E. Cates, J. Fluid Mech. 847, 700 (2018).

[35] S. F. Edwards and R. B. S. Oakeshott, Physica (Amsterdam) 157A, 1080 (1989).

[36] S. Martiniani, K. J. Schrenk, K. Ramola, B. Chakraborty, and D. Frenkel, Nat. Phys. 13, 848 (2017).

[37] A. Baule, F. Morone, H. J. Herrmann, and H. A. Makse, Rev. Mod. Phys. 90, 015006 (2018). 